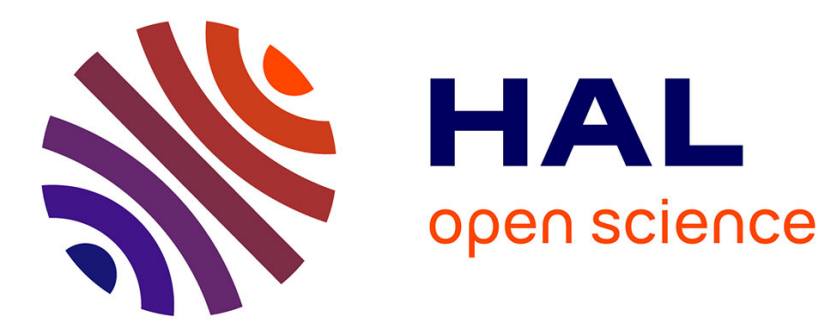

\title{
Le symposium international du Val d'Aoste sur les avalanches
}

Louis de Crécy

\section{To cite this version:}

Louis de Crécy. Le symposium international du Val d'Aoste sur les avalanches. Revue forestière française, 1975, 27 (3), pp.243-245. 10.4267/2042/20943 . hal-03395527

\section{HAL Id: hal-03395527 \\ https://hal.science/hal-03395527}

Submitted on 22 Oct 2021

HAL is a multi-disciplinary open access archive for the deposit and dissemination of scientific research documents, whether they are published or not. The documents may come from teaching and research institutions in France or abroad, or from public or private research centers.
L'archive ouverte pluridisciplinaire HAL, est destinée au dépôt et à la diffusion de documents scientifiques de niveau recherche, publiés ou non, émanant des établissements d'enseignement et de recherche français ou étrangers, des laboratoires publics ou privés. 


\title{
LE SYMPOSIUM INTERNATIONAL DU VAL D'AOSTE SUR LES AVALANCHES
}

\author{
L. de CRÉCY
}

Class. Oxford $116.7: 971$

A l'initiative de la Région autonome du Val d'Aoste, s'est tenu du 23 au 26 février 1975 à Saint-Vincentd'Aoste (Italie) un symposium international sur le thème : "les avalanches : nouvelles techniques des systèmes de prévention et de défense $"$.

Les courts délais entre la convocation et la tenue de cette réunion internationale expliquent que les pays éloignés de l'Arc Alpin n'aient pas pu assurer une participation active. Seuls, l'Allemagne, l'Autriche, la France, l'Italie, la Norvège et la Suisse ont présenté des communications.

L'étendue même du thème proposé permettait de faire le point sur l'état de développement de la lutte préventive contre l'avalanche dans les différents pays présents.

Une première constatation s'impose : l'écart technologique entre les différents pays qui était flagrant il y a 5 ou 10 ans, tend à s'estomper. Les Suisses d'abord, puis les Autrichiens disposaient il y a 5 à 10 ans encore d'une avance technologique et scientifique très considérable. Sous la pression du développement du ski et, hélas, sous celle d'accidents répétés, les autres pays alpins ont consenti des efforts considérables qui leur ont permis de rattraper une bonne partie de leur retard.

L'autre constatation est la similitude des préoccupations à l'ordre du jour dans les différents pays : mises à part les techniques de sauvetage et de recherche de victimes qui n'entraient pas dans le cadre du symposium, la lutte préventive contre l'avalanche s'organise dans tous les pays selon les quatre grands axes logiques suivants:

- La prévision, par la voie de bulletins météorologiques, du danger d’avalanches : les méthodes d'élaboration sont semblables. Mais on discute l'opportunité de diffuser largement auprès du grand public tous les bulletins ou de réserver les plus techniques d'entre eux aux seuls responsables suffisamment avertis pour les interpréter correctement. A travers les diverses solutions adoptées apparait le problème de responsabilité du prévisionniste ainsi que celui, de plus en plus angoissant, de la sécurité des skieurs hors pistes (randonneurs de "ski de printemps").

- La «localisation des avalanches" préoccupe également tous les pays, essentiellement en vue de déterminer l'étendue des espaces constructibles en montagne, infiniment plus réduits qu'on ne l'imagine souvent.

L'échelle adoptée est celle du plan d'urbanisme (1/1 000 à 1/5000); le zonage comprend au minimum trois couleurs, une zone "douteuse» où il faut prévoir si l'on veut construire, une gamme variée de précautions spéciales de différents types s'intercalant obligatoirement entre la zone reconnue dangereuse et la zone présumée sûre. La rédaction de tels documents est toujours longue et coûteuse, les méthodes 
sont encore incertaines : enquêtes auprès des habitants, consultations des archives locales, "calculs » plus ou moins entachés d'empirisme, observation soignée du terrain.

De tels "Plans des Zones Exposées aux Avalanches" sont également entrepris en France, mais notre pays a adopté une étape intermédiaire qui a suscité un grand intérêt chez nos voisins en réalisant " la carte de localisation probable des avalanches".

L'originalité de cette carte réside :

- dans ses méthodes : à la traditionnelle enquête auprès des habitants et dans les archives s'ajoute une photo-interprétation systématique des couvertures aériennes d'été permettant de relever les détails topographiques et géomorphologiques ou les traces sur la couverture végétale qui peuvent indiquer un ancien passage d'avalanche:

- dans son éche/le : le 1/20 000 ne convient pas à l'architecte, mais il suffit à l'« aménageur du territoire " qui a besoin d'un document rapide et pas trop coûteux à établir ( 500000 ha parcourus en 5 ans en France pour un peu plus de $7 \mathrm{~F} / \mathrm{ha}$ ), susceptible d'être utilisé même dans les zones dépourvues d'établissement humain hivernal ancien (grâce à la photo-interprétation), même si les renseignements obtenus ne sont pas d'une "fiabilité " absolue;

- dans sa conception même : elle ne prétend représenter que ce qui est connu des avalanches qui ont eu lieu dans le passé sans viser à une étude prospective. Elle peut donc s'affranchir, surtout au $1 / 20000$, de la «zone douteuse ».

- Les techniques de déc/enchements préventifs suscitent de plus en plus d'intérêt auprès des stations de ski, que ce soit pour les voies d'accès, ou pour les pistes de ski. Mais elles se heurtent à de redoutables problèmes réglementaires (emploi des explosifs) ou juridiques (responsabilité en cas de dégâts provoqués).

- Le génie paravalanche est désormais classique. En défense active, le débat ne porte plus sur l'efficacité des ouvrages modernes à claire-voie qui est partout reconnue, mais sur le choix du type d'ouvrage (claie, râtelier, filet), sur leur conception mécanique (articulation), sur leur mise en place (transport, ancrage), sur les matériaux à utiliser (place des alliages d'aluminium et des fibres synthétiques en face de l'acier et du béton), sur leur disposition (un consensus de plus en plus large s'exprime pour les lignes continues) ou leur prix (variable mais unanimement reconnu exorbitant). Le rôle de la forêt est volontiers reconnu par tous mais les études de reboisement en haute altitude ne sont sérieusement poussées qu'en Autriche et en Suisse.

Le rôle des ouvrages déflecteurs modifiant le dépôt de la neige sous l'effet du vent est admis mais les règles d'emploi de ces constructions sont loin d'être parfaitement élucidées et l'empirisme qui règne en la matière préoccupe les chercheurs.

En matière de défense passive, on est également encore mal renseigné sur les efforts que doivent encaisser les différents types d'ouvrages de la part des avalanches en mouvement. La recherche sur la dynamique des avalanches peut seule apporter des éléments rationnels.

Un trait constant qui ressort des exposés généraux présentés à Saint-Vincent-d'Aoste est un appel à la puissance publique pour intervenir dans ce domaine nouveau : le pouvoir exécutif est sollicité pour organiser et financer la lutte, le pouvoir législatif pour permettre l'emploi des méthodes nouvelles, le pouvoir judiciaire enfin doit aider à la mise en place d'une sécurité maximum en assouplissant certaines jurisprudences en matière de responsabilité.

Les progrès scientifiques et techniques accomplis récemment ont en effet considérablement élargi la gamme des moyens de lutte disponibles contre l'avalanche et leur efficacité. Mais le coût de leur mise en œuvre et en particulier celui du génie paravalanche est d'un ordre de grandeur dont les gouvernements n'ont pas encore pris l'exacte mesure.

Le cadre réglementaire et législatif présente également certaines lacunes, par exemple pour l'emploi des explosifs dans le déclenchement préventif des avalanches ou le moyen d'assurer l'entretien des ouvrages ou forêts de protection à l'abri desquels on a donné un permis de construire.

Mais ce qui inquiète surtout les animateurs de la lutte contre les avalanches, ce sont les responsabilités qu'ils encourent lorsqu'ils agissent. Tant que le fléau était réputé imprévisible et imparable, on pouvait invoquer le cas de force majeure. Mais dès que quelqu'un s'est avisé de baliser une piste de ski, de construire un ouvrage de protection, de déclencher préventivement une avalanche, de prévoir ou de cartographier le risque d'avalanche, il a, aux yeux des juristes, apporté la preuve que le danger était prévisible ou parable et qu'il y a donc lieu, en cas d'accident, de rechercher sa responsabilité civile ou pénale. Or, même si la mise en œuvre des moyens modernes de lutte réduit dans des proportions énormes 
la probabilité d'un accident, il ne serait pas scientifique d'affirmer qu'elle l'annule complètement. II subsistera toujours, si faible soit-elle, la probabilité d'une erreur que la jurisprudence, dans le souci de trouver un patrimoine capable d'indemniser les victimes, qualifie de faute. A défaut, pour les facultés de droit, d'introduire dans leurs programmes l'étude de la notion de probabilité et d'en tirer les conséquences dans l'élaboration de leurs constructions juridiques, elles risquent d'entretenir avec les scientifiques des malentendus qui peuvent être paralysants et s'opposer gravement à bien des progrès dans l'organisation de la sécurité publique. Cette inquiétude est vivement ressentie à tous les niveaux et dans tous les pays de l'Arc Alpin parmi les spécialistes de la défense contre l'avalanche.

En dehors des communications techniques qui avaient pour but de faire connaittre les méthodes en usage dans les différents pays participants, un certain nombre d'auteurs ont rendu compte de leurs recherches dans des domaines scientifiques plus avancés. Nouvelles méthodes simplifiées de restitution de photos aériennes exposées par un chercheur italien, différents essais de simulation d'écoulement d'avalanche sur maquettes ou en canal noyé, ou de vent sur des barrières à neige en soufflerie, des mesures directes de paramètres (vitesse, débit, densité, pression sur un obstacle...) d'une avalanche en mouvement déclenchée artificiellement, telles sont les principales activités de recherche qui ont retenu l'attention des techniciens.

Qu'il me soit permis de souligner à cet égard l'importance et l'intérêt de la contribution française (12 communications sur 23) notamment dans le domaine scientifique. L'effort fourni par la France depuis 1970 commence à porter ses fruits et à mettre notre pays " dans la course " dans le domaine de la lutte contre I'avalanche. Beaucoup de nos voisins ont apprécié, et certains nous envient, la souplesse et l'efficacité de la structure de concertation et de vulgarisation dont s'est dotée la France en 1971 en créant I'Association nationale pour l'étude de la neige et des avalanches.

Il reste, pour terminer, à remercier nos hôtes de l'opulente Région autonome du Val d'Aoste pour leur somptueuse réception et la parfaite organisation de ce symposium et de la très sympathique journée sur le terrain qui l'a suivi, sur les pistes de Courmayeur. La nombreuse délégation française était là en voisine chez des cousins très proches par la langue et par les mœurs. Beaucoup ont souhaité que l'initiative Valdotaine ait une suite et espèrent que des réunions du même type aient lieu périodiquement dans l'avenir pour faire le point de l'avancement des techniques en matière de lutte contre l'avalanche.

\section{Louis de CRÉCY}

Ingénieur en chef du G.R.E.F.

Chef de la Division "Nivologie" CENTRE TECHNIQUE DU GÉNIE RURAL DES EAUX ET DES FORETS Groupement de Grenoble B.P. 114 38402 SAINT-MARTIN-D'HÉRES 